\title{
Multi-targets localization based on ARMA and GA in WSNs
}

\author{
Heng Zhang*, and Zhongming Pan \\ College of Artificial Intelligence, National University of Defense Technology, Changsha, 410073, \\ China
}

\begin{abstract}
Multi-target localization methods for locating of the movingtarget in interested area monitored by Wireless Sensor Networks (WSNs) are nowadays a popular subject of study. The methods can be classified into two categories: range-free algorithm and range-based algorithm. In this work, we propose a novel multi-target localization method, which belongs to the category of range-based algorithm, by using a genetic algorithm (GA) for searching optimal solution of the objective function of multi-target localization. The objective function is only a group of linear equations with independent variables of acoustic energies calculated at each sensor-node in a WSN. However, application of the method, the accuracy of multi-target localization is sensitive to the SNR of the measured sound signals at each node, thus a denoising strategy should be inserted into the method. It turned out that the measured sound noise, comparing intrinsic sensor noise and environmental noise, may be considered as an Autoregressive Moving Average (ARMA) process. Thus, by building the ARMA model, the noise sequence commingled with the target signals can be predicted. As a consequence, the power of the noises can be subtracted from the measured sound signals for revealing the target signal's power. The results in present work demonstrate the advantage of the proposed method.
\end{abstract}

\section{Introductions}

WSNs consists of nodes with capabilities of sensing, signal processing and communicating. In addition to object detecting, one is also interested in the inherent property of control and activation in WSNs[1]. One of the most significant application of WSNs is moving targets localization in the interested area, which is the preconditions for targets tracking in military operation. Target (signal source) localization algorithms applied to WSNs can be classified into two categories: range-free algorithm and range-based algorithm. The former requires special hardware to deal with the radio signal intensity for calculating target position, while no extra hardware is required in the latter [2].

Aiming at achieving accurate localization of the acoustic source and fast convergence, Xia $\mathrm{Na}$ [3] proposed a simultaneous perturbation stochastic approximation (SPSA)-based solution by computing the approximate the gradient of the target function, and an island

\footnotetext{
* Corresponding author: zhnudt@126.com
} 
model using Voronoi diagram to reduce the searching space. Jeongkeun Lee et al [4] proposed a novel localization algorithm of a target named as Ratiometric Vector Iteration (RVI), which can locate the target accurately with only three sensors' participation by iteratively updating the estimated position according to the principle of the ratio telemeter. However, as for multi-target localization, RVI algorithm brings forth a new difficulty for processing the correlative random data. Another approach for target localization is maximum likelihood (ML) estimation method [5] based on acoustic power of individual sensor-node. Comparing to the existing source localization methods, the ML estimation method can achieve more accurate results and enhance the capability of multiple source localization [6], but it is sensitive to the associated parameters. M. Ziaur Rahman et al [7] presented a method known as passive source localization (PSL). Application of the PSL method, the probabilistic position of the multi-target can be estimated by analysing the power spectral density (PSD) of the signal at each node, while the accuracy of multi-target localization can be insured by making a statistical decision fusion of the estimator at each control node intercalated in the WSN. All of the methods mentioned above are belonged to the category of range-free algorithm.

Just as its name implies, the range-based localization algorithms, such as time delay of arrival ( TDOA) [8], direction of arrival (DOA) [9], and received signal strength (RSS) indicator, use the absolute distances between the signal sources and the neighboring nodes in WSNs to estimate the target position. After the distances being estimated, the position of the target can be calculating by trilateration or multilateration algorithm. $\mathrm{Zu} \mathrm{Linan[8]}$ presented an algorithm for multi-target localization based on the TDOA algorithm under the condition that the multi-sound sources is freed from the environmental noises, but it is not suitable for multi-target localization in WSNs for it requires real-time communicating between the targets and the nodes, which is impracticable in the WSN of non-cooperative function.

The rest of the paper is organized as follows: In section 3, the ARMA model of noise process is built to estimated L-step noise sequence mixed together with the measured sound signals. In Section 4, GA is introduced to get the optimal solution of the objective function of multi-target localization. In section 5, the numerical simulations of noise power predictor based on ARMA model, as well as the multi-target localization based on RSS and GA are presented in Section 5 to validate our proposed method, the data used in the simulation is reference to bibliography [10]. Section 6 concludes the paper.

\section{Multi-target model}

\subsection{Sensing model}

The localization of multi-target algorithm based on RSS implies the property that sound power attenuates with the square of the distance as it transmitting in the air. Assumed there are $\mathrm{n}$ sensor-nodes and $\mathrm{m}$ targets in the area of interest (AOI). As presented in bibliography [11], the power of the acoustic signal originated by the $j$ th target source at the ith sensornode is:

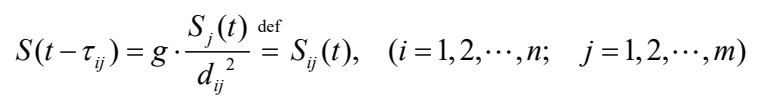

where $S_{j}(t)$ represents the acoustic power of the $j$ th target source, $S\left(t-\tau_{i j}\right)$ the acoustic power of the $i$ th sensor-node. And $g$ is attenuation coefficient (let $g=1$ for simplifying), $d_{i j}$ the distance between the $j$ th target source and the $i$ th sensor-node, and $\tau_{i j}$ the time delay of sound wave transmitted from the $j$ th target source to the $i$ th sensor-node. 
In this paper, we assume that every sensor-node is equipped with a microphone for sensing sound waves, and an accurate synchronizing time of all sensor-nodes is insured.

\subsection{Multi-target localization model}

Let the output signal of the $i$ th sensor-node to be $z_{i}(t)$, then the power of the measured acoustic signal zi $(t)$ can be expressed as

$$
W_{i}(t)=E\left[z_{i}^{2}(t)\right]=W_{i}(t)=\sum_{j=1}^{m} S_{i j}(t), \quad(i=1,2, \cdots, n)
$$

As shown in [12] and figure 1, the acoustic power sensor-node $i$ received is composed of several attenuated signals generated by the multi-target source. The acoustic power of $i$ th sensor-node can be calculated as follows

Substituting equation (1) into equation (2), we get

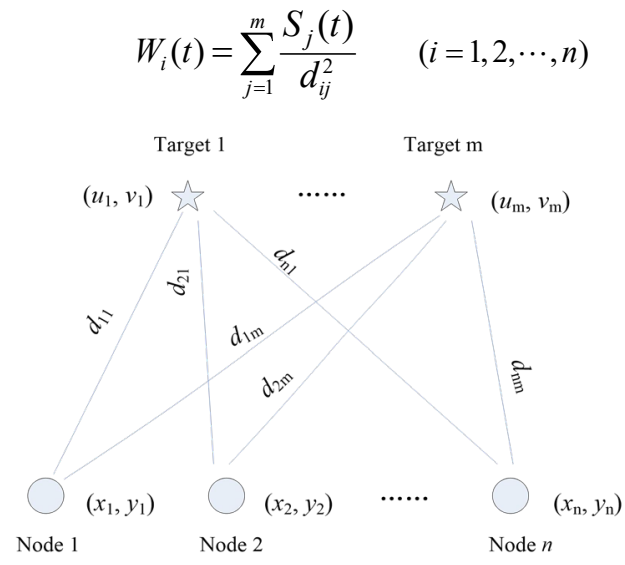

Fig. 1. Sensor-nodes Measurements of multi-target acoustic power.

Supposing the placement of the target and the sensor-node to be on the horizontal plane, then, we have

$$
d_{i j}=\left(x_{i}-u_{j}\right)^{2}+\left(y_{i}-v_{j}\right)^{2}, \quad(i=1,2, \cdots, n ; \quad j=1,2, \cdots, m)
$$

where, $\left(u_{j}, v_{j}\right)$ is the unknown coordinate of target $j$, and $\left(x_{i}, y_{i}\right)$ the known coordinate of sensor-node $i$.

Now the problem dealt with is how to determine the unknown variables $\left(u_{j}, v_{j}\right)$ and $S_{j}(t)$. The multiple variables made it difficult to solve equation (3) directly. So we transformed the target localization problem into an optimal estimation problem. The objective function of the optimal estimation problem can be expressed as:

$$
f\left(u^{*}, v^{*}, S^{*}\right)=\sum_{i=1}^{n}\left|W_{i}(t)-\sum_{j=1}^{m} \frac{S_{j}^{*}(t)}{d_{i j}^{* 2}}\right|
$$

where

$$
d_{i j}^{*}=\left(\left(x_{i}-u_{j}^{*}\right)^{2}+\left(y_{i}-v_{j}^{*}\right)^{2}\right)^{1 / 2}, \quad(i=1,2, \cdots, n ; \quad j=1,2, \cdots, m)
$$

and $\left(u_{j}^{*}, v_{j}^{*}\right)$ denotes the tentative coordinate of $\left(u_{j}, v_{j}\right), S_{j}^{*}(t)$ the tentative value of $S_{j}(t)$.

As for the target localization problem, if there are q targets trespassing in AOI at the same time, then it requires that the power signals of $p$ sensors $(p \geq q+2)$ are greater than the preassigned threshold $\lambda$. When all the tentative values are approximate to actual values, the objective function $f\left(u^{*}, v^{*}, S^{*}\right)$ is approaching to zero. 


\section{Noise prediction using ARMA Model}

\subsection{ARMA model}

Assumed that time series $\left\{n_{k-1}, n_{k-2}, \ldots, n_{k-p}\right\}$ is the sensor measurements sequence of the environment noise. $n_{k}$ can be described by ARMA model as follows:

$$
n_{k}=a_{1} n_{k-1}+a_{2} n_{k-2}+\cdots+a_{p} n_{k-p}+e_{k}-b_{1} e_{k-1}-b_{2} e_{k-2}-\cdots-b_{q} e_{k-q}
$$

where $a_{i}(i=1,2, \ldots, p)$ is the AR parameter; $b_{l}(l=1,2, \ldots, p)$ is the MA parameter; $e_{k}$ is the Gaussian white noise with zero means and a variance of $\sigma_{e}{ }^{2}$. In this study, the $\operatorname{ARMA}(4,3)$ model was selected due to its simplicity and its observed good fit to the data.

\subsection{L-step prediction}

Denote the environment noise sequence as $\left\{n_{k}, n_{k-1}, \ldots, n_{k-p}\right\}$. The prediction of the noise sequences at moment $k+L$ is noted as $\hat{n}_{k+L \mid k}(L=1,2, \ldots)$.

As shown in [12], equation (7) can be rewritten as

$$
A(z) n_{k}=\gamma B(z) e_{k} ; \quad A(z)=1-a_{1} z^{-1}-\cdots-a_{p} z^{-p}, \quad B(z)=1-b_{1} z^{-1}-\cdots-b_{q} z^{-q}
$$

where $\gamma$ is a constant, $n_{k}$ is zero mean random process, $e_{k}$ sequence of white Gaussian noise, and $E\left[x_{k} e_{k+L}\right]=0$

Multiplied with the advance shift operator $z^{L}$, equation (8) can be expressed as follows:

$$
n_{k+L}=\frac{B(z)}{A(z)} \gamma z^{L} e_{k}=\left[F(z)+\frac{z^{-L} G(z)}{A(z)}\right] \gamma z^{L} e_{k}=\gamma F(z) e_{k+L}+\frac{G(z)}{A(z)} \gamma e_{k}
$$

Multinomial $F(z)$ is the quotient of $B(z)$ divided by $A(z)$, and $z^{-L} G(z)$ the remainder of the division, $\hat{n}_{k+L \mid k}$ the least mean squares (LMS) estimation of $L$-step prediction based on equation (8). Then the $L$-step prediction is:

$$
\hat{n}_{k+L \mid k}=\frac{G(z)}{B(z)} n_{k}=-b_{1} \hat{n}_{k+L-1 \mid k-1}-b_{2} \hat{n}_{k+L-2 \mid k-2}-\cdots-b_{q} \hat{n}_{k+L-q \mid k-q}+g_{0} n_{k}+\cdots+g_{p-1} n_{k-p+1}
$$

\section{Multi-target localization based on Genetic Algorithm}

In this work, GA is chosen for solving the optimal estimation problem, because it can handle any kind of objective functions and any kind of constraint conditions without analytic representations of the optimization problems. In GA, the variables $u^{*}, v^{*}$ and $S^{*}$ are represented as genes in a chromosome which is evaluated according to its fitness. The fitness function is defined as

$$
\operatorname{fit}\left(u^{*}, v^{*}, S^{*}\right)=\frac{1}{1+f\left(u^{*}, v^{*}, S^{*}\right)}
$$

The algorithmic routine starts with a set of assumed solution $u^{*}, v^{*}$ and $S^{*}$, which is encoded as a set of chromosomes priorly. The initial population of chromosomes is selected randomly. After the population being operated by the genetic operators, such as selection, mutation and crossover, those with better fitness are selected as next generation of the chromosomes. The repetitionary operation of the population of chromosomes continues until the fitness function is approached to the preappointed value, as shown in figure 2. 


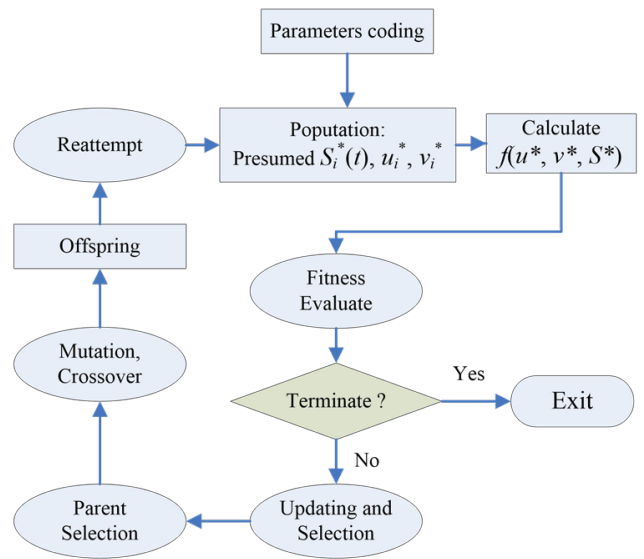

Fig. 2. Flowchart of Genetic algorithm.

\subsection{Coding schemes}

Each chromosome is usually coded as a fixed-length binary string. The length of the string depends on the variables being searching and the required precision. Assumed the domain of variable is $\left[U_{1}, U_{2}\right]$ and the precision requirement is $j$ places after the decimal point, then the domain $\left[U_{1}, U_{2}\right]$ should be divided into $M$ equal size ranges, that is

$$
M=\left(U_{2}-U_{1}\right) \times 10^{j}
$$

$l$ is the length of binary code string and is determined by following equation:

$$
2^{l-1} \leq M \leq 2^{l}
$$

The binary string composed of the variables is regarded as a chromosome. According to the objective function mentioned above, the variables are expressed as

$$
\left[u_{1}^{*}, u_{2}^{*}, \cdots u_{m}^{*}\left|v_{1}^{*}, v_{2}^{*}, \cdots v_{m}^{*}\right| S_{1}^{*}, S_{2}^{*}, \cdots S_{m}^{*}\right]
$$

The chromosome shown in figure 3 is corresponding to a group of optimized variables, where $p, q$ and $r$ are length of the binary code of $u_{j}^{*}, v_{j}^{*}$ and $S_{j}^{*}$ respectively.

Chromosome: 9 Part 1
\begin{tabular}{|c|c|c|c|c|c|c|c|c|}
\hline \multicolumn{3}{|c|}{$u_{1}{ }^{*}$} & $\cdots$ & \multicolumn{3}{|c|}{$u_{m}{ }^{*}$} \\
\hline$b_{1}$ & $b_{2}$ & $\cdots$ & $b_{p}$ & $\cdots$ & $b_{1}$ & $b_{2}$ & $\cdots$ & $b_{p}$ \\
\hline \multicolumn{10}{|c|}{ Part 3} \\
\hline$b_{1}$ & $b_{2}$ & $\cdots$ & $b_{r}$ & $\cdots$ & $b_{1}$ & $b_{2}$ & $\cdots$ & $b_{r}$ \\
\hline
\end{tabular}

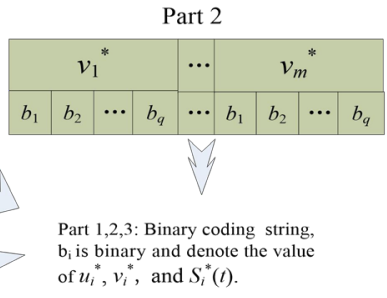

Fig. 3. Chromosome using binary coding.

\subsection{Genetic Operators}

There are three basic genetic operators in GA, which are selection, crossover and mutation.

Selection: The selection operator is an artificial version of natural selection to create population of chromosomes from one generation to next generation, and the chromosomes in the present population with better fitness value have higher probabilities of being selected as next generation [13]. 
Crossover: New chromosomes (children) are generally created as offspring of two parents, as shown in figure 4 . The crossover points are randomly selected within the chromosome of each parent. The parts delimited by the crossover points are then interchanged between the parents [14].

Mutation: A new individual is created by making modifications to one selected individual. The modifications can consist of changing one or more values in the representation or in adding / deleting parts of the representation.

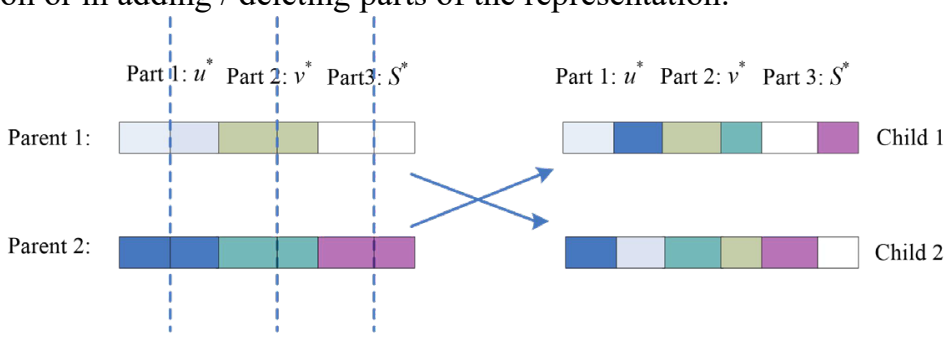

Fig. 4. Crossover of GA.

\section{Simulation}

The purpose of GA is to searching the minimum value of the objective function under the constraint condition, that is

$$
\left\{\begin{array}{l}
\min f\left(u^{*}, v^{*}, S^{*}\right) \\
W_{i}(t) \geq \lambda
\end{array} \quad(i=1,2, \cdots, n ; \quad j=1,2, \cdots, m)\right.
$$

However, the noise power mixed with $W_{i}(t)$ is found to be rather effective on the precision of the targets localization, therefore, the noise power should be eliminated. For this purpose, an ARMA model of noise sequence is established for predicting the aftertime noise sequence described in equation (10). The chart of the denoising procedure based on ARMA model is shown in figure 5. In which, operator $D$ represents first order differential action of the sampled sequence of noise (reference to bibliography [10]) since it is not stationary, and $D^{-1}$ the invert operator of $D$. we will discuss this issue in detail in next section.

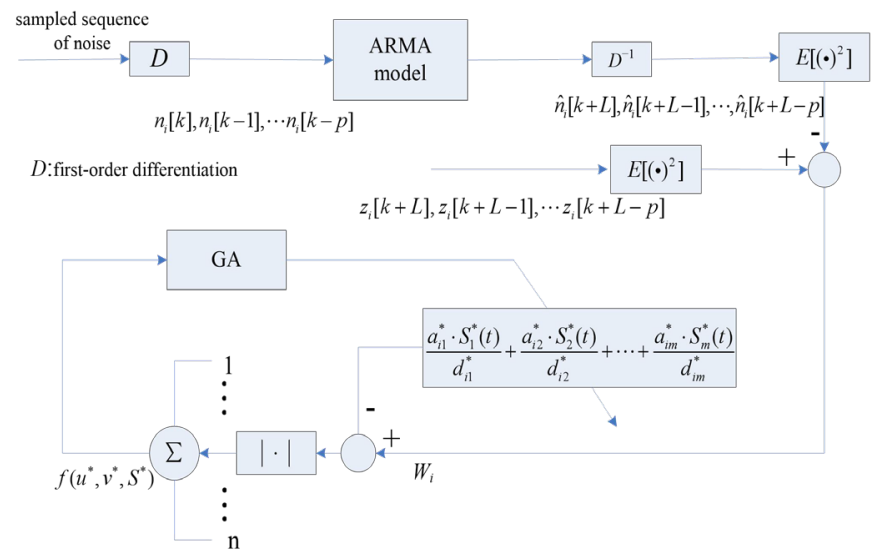

Fig. 5. Multi-targets localization Model.

In the figure above $a_{i m}{ }^{*}$ is attenuation coefficient (let $a_{i m}{ }^{*}=1$ for simplifying). Assumed the length of the sampled data of noise is $p+1$, then the output sequence of sensor-node $i$ at 
moment $k$ can be written as $\mathrm{s} \__{-} n_{i}[k], \mathrm{s} \_n_{i}[k-1], \ldots, s_{-} n_{i}[k-p]$, and the first order differential of the sequence denoted as $n_{i}[k], n_{i}[k-1], \ldots, n_{i}[k-p]$. As consequence, the L-step prediction of the sequence of based on ARMA model is $n_{i}^{\wedge}[k+L], n_{i}^{\wedge}[k+L-1], \ldots, n_{i}[k+L-p]$. The invert of the first order differential of the predicted sequence denoted as $\tilde{n}_{i}[k+L], \tilde{n}_{i}[k+L-1], \ldots$, $\tilde{n}_{i}[k+L-p]$. The sequence $z_{i}[k+L], z_{i}[k+L-1], \ldots, z_{i}[k+L-p]$ represents the measured sound signal at moment $k+L$, thus, the power of the measured sound signal after denoising can be expressed as

$$
W_{i}[k+L-l]=E\left\{z_{i}^{2}[k+L-l]\right\}-E\left\{\hat{n}_{i}^{2}[k+L-l]\right\}, \quad(l=0,1, \cdots, p)
$$

\subsection{Noise prediction based on ARMA model}

Differentiation is an easy but effective way to implement stabilizing of the time series when it is not stationary. The original time series and its first-order differentiation sequence, with their autocorrelation function and partial correlation function are shown in figure 6 . From the autocorrelation curve of the original sequence at the first row in figure 6 , we can conclude that the original sequence is not white noise sequence. Thus, we can't build ARMA model for the sequence. However, the autocorrelation curve of the first-order differentiation of the original noise sequence at the second row in figure 6 is approximate to $\delta$ - function, but it is tail off, and the partial correlation curve is the same. It is showed good compatibility of the first-order differentiation of the original sequence to an ARMA $(4,3)$ model.
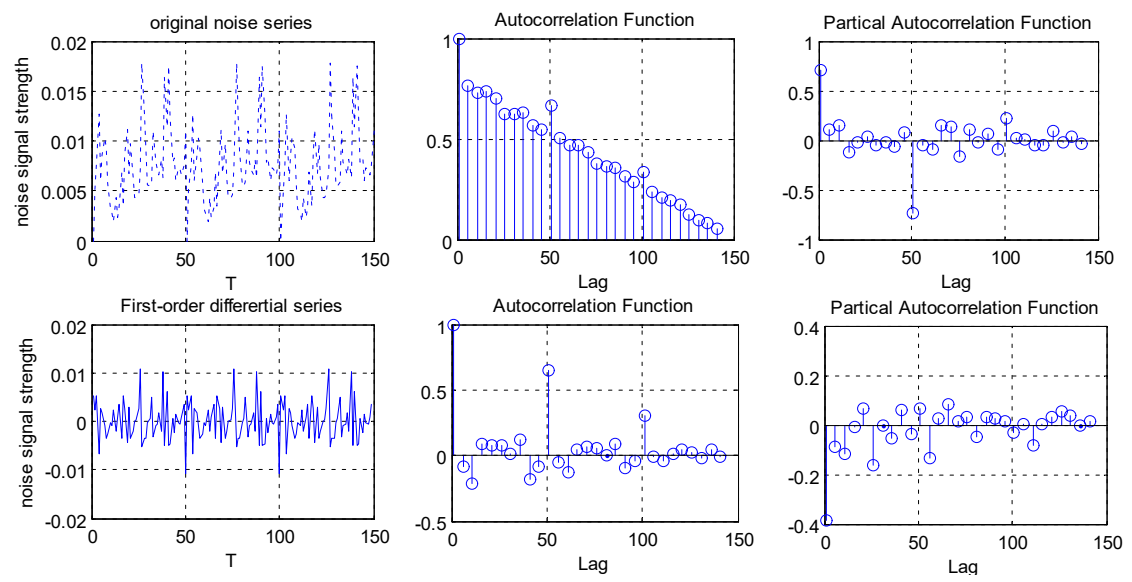

Fig. 6. Noise series, autocorrelation function, and partial autocorrelation function.

Figure 7 shows the original noise sequence and the invert operating $\left(\mathrm{D}^{-1}\right)$ of the L-step $(L=3)$ prediction data based on the ARMA $(4,3)$ model, respectively. We can see that the two curves are quite approximative.

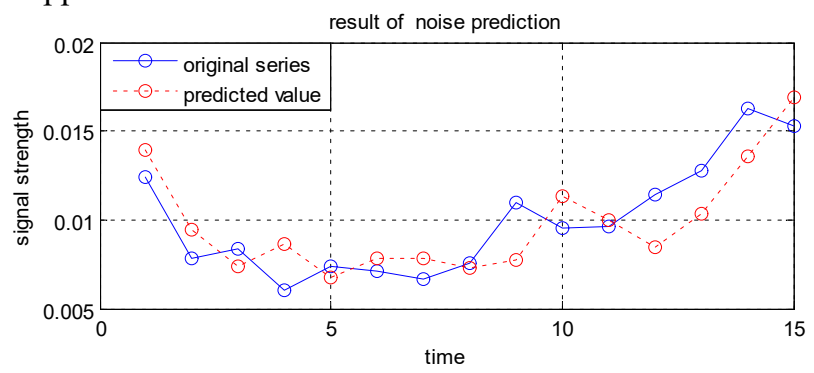

Fig. 7. Result of noise prediction. 


\subsection{Multi-target localization using genetic algorithm (GA)}

There are five sensor-nodes and one target configurated a map as shown at the left of first low in figure 8. Considering the power levels generated by civil or military vehicle, the signal strength of the target is supposed to be $80 \mathrm{~dB}$. The L-step prediction data which is magnified 100 times, is added to the targets' acoustic signal to simulate the external noise.

Figure 8 shows the located positions while the numbers of working sensor-nodes are 3, 4 and 5 respectively. The right of the first low in figure 8 are the cases with no external noise mixed in the sampled sound signal; the left of the second low the cases with external noises mixed in the sampled sound signal; and the right of the second low the cases with denosing procedure based on $\operatorname{AMMA}(4,3)$ model. Figure 9 shows the average localization errors in the cases mentioned above. Apparently, the localization errors in the cases of considering the effect of a noise disturbance are bigger than that in the other cases, and the proposed denosing method using ARMA model can reduce the effectiveness of noise jamming. Furthermore, the more number of sensor-nodes are apperceiving the target signal simultaneously, the more accurate positioning can be achieved.
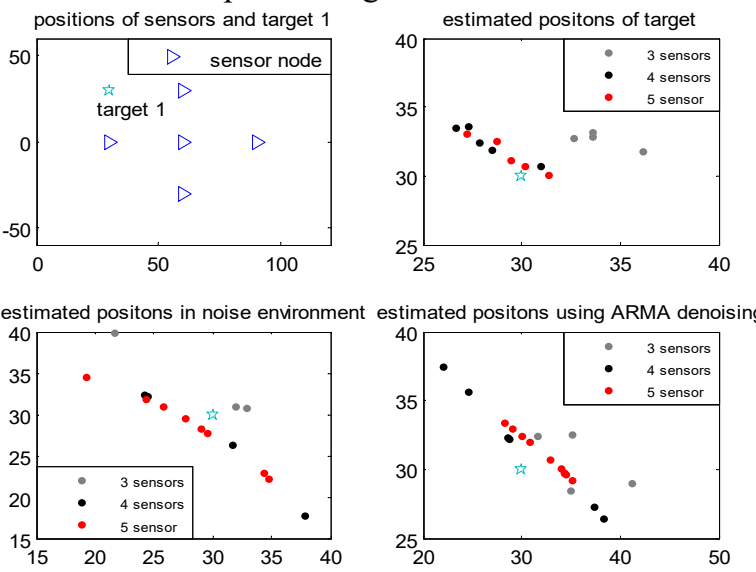

Fig. 8. The results of single target localization.

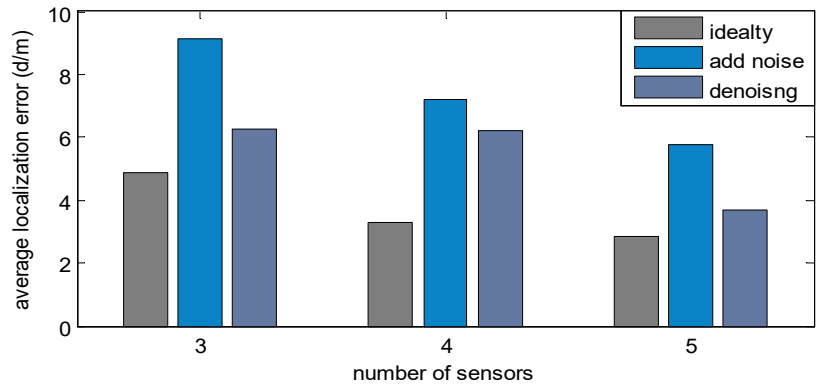

Fig. 9. Single-target localization errors.

Another simulation was conducted using two acoustic sources, the signal source energy are $60 \mathrm{~dB}$ and $80 \mathrm{~dB}$ respectively. There are five sensor-nodes and two targets configurated a map as shown at the left of first low in figure 10. The middle of the first low in figure 10 shows the located positions in the cases with only 4 working sensors; while the right shows the located positions in the cases with 5 working sensors.

As indicated in figure 11, the more number of sensor-nodes are apperceiving the target signal simultaneously, the more accurate positioning can be achieved. Beside this, it also shows that the proposed method is suitable for multi-target localization. 

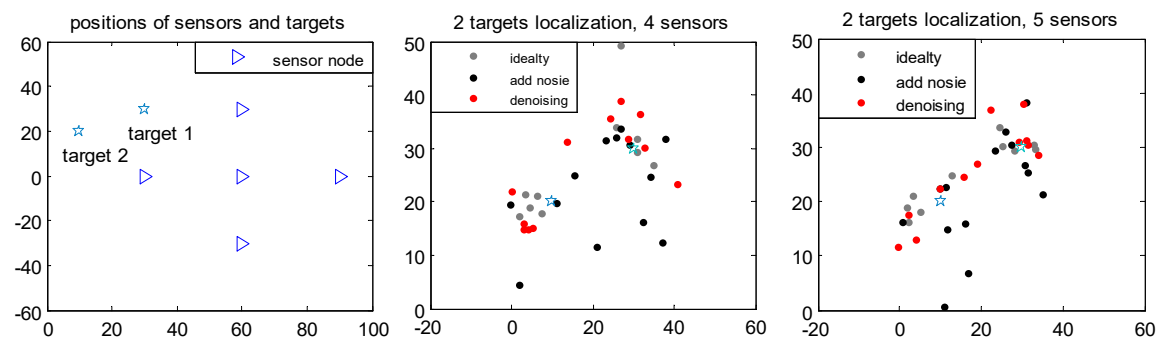

Fig. 10. Two targets localization.

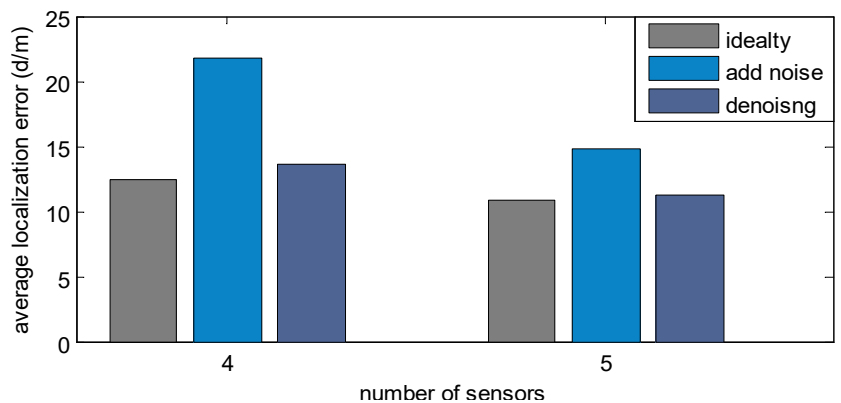

Fig. 11. Two targets localization errors.

We can draw a conclusion intuitively that the more sensor-nodes deployed in a WSN, the more constraint conditions in the objective function should be satisfied, which may help GA to search the optimal position more accurate.

\section{Conclusion}

We have introduced a novel strategy to locate multi-target in the detecting range of a WSN. We propose to use the ARMA model for predicting the noise mixed with the measured sound signals in order to denoise at the moment of sampling. Simulation results indicate that for any kind of noise which can be whitened, the performance of the proposed method was found to be superiority to the multi-target localization algorithm based on RSS and GA.

The proposed method is still applicable when acoustic sensor was replaced by magnetic sensor, since the intensity of magnetic anomaly caused by the target may be considered as dipole field. As is well known, a WSN with acoustic sensors or magnetic sensors is experiencing a plethora of applications nowadays.

\section{References}

1. Kazem Sohraby, Daniel Minoli, Taieb Znati 2007 Wireless sensor networks: technology, protocols, and applications. (New Jersey: John Wiley \& Sons) pp 1

2. Patrik Moravek, Dan Komosky Milan Simek, David Gribau, AntonioLazaro 2011 Radio Engineering. 20937

3. Xia Na, Du Huazheng, Li Shuangjiang 2013 Ad Hoc \& Sensor Wireless Networks. 19 277

4. Jeongkeun Lee, Kideok Cho, Seungjae Lee 2006 Computer Communications. 292494

5. Raida Al Alawi 2011 RSSI Based Location Estimation in Wireless Sensors Networks, $17^{\text {th }}$ IEEE International Conference on Networks. 118 
6. X. Sheng, Y. H. Hu 2004 Sequential acoustic power based source localization using particle filter in a distributed sensor network. International Conference on Acoustics, Speech, and Signal Processing, 972

7. M. Ziaur Rahman, Gour C. Karmakar, Laurence S, Dooley 2005 Passive Source Localization using Power Spectral Analysis and Decision Fusion in Wireless Distributed Sensor Networks, Proceedings of the International Conference on Information Technology 260

8. Zu Linan, Yang Peng, Sun Hao, Chen Lingling 2010 Sound Source Target Localization System of Mobile Robot. $11^{\text {th }}$ International Conference on Control Automation Robotics and Vision 2289

9. N. Iwakiri, T. Kobayashi 2008 Journal of Communications 312

10. Marco F. Duarte, Yu Hen Hu 2004 Journal of Parallel and Distributed Computing 64 826

11. Pan Zhongming 2012 Analysis of Random Signal and Optimal Estimation Method. (Changsha: Defense Industry)

12. Yang Y, Blum R S, Sadler B 2009 IEEE Transactions on Signal Processing 572050

13. Zne-Jung Lee 2008 Expert Systems with Applications 34384

14. Gabor Rennera, Aniko Ekarta 2003 Computer Aided Design 35709 